\title{
Human Computer Interactions for Amyotrophic Lateral Sclerosis Patients
}

\author{
Ali Bülent Uşaklı \\ The NCO Academy \\ Turkey
}

\section{Introduction}

In this chapter, alternative communication and device control channels, which are helpful for Amyotrophic lateral sclerosis (ALS) patients, are introduced. In this context, human computer interactions (HCIs) will be discussed in three respects; electrical brain activities, eye movements and hemoglobin level in the blood.

With technological advances, fighting or minimization side effects of the diseases is the main purpose of biomedical research. Under this motto, this chapter focuses on HCIs for individuals suffering from motor neuron diseases. ALS is a progressive neurodegenerative disease caused by the degeneration of motor neurons. ALS or other tetraplegic clinical conditions, otherwise known as the locked-in syndrome, have severe disabilities in controlling muscles and consequently have problems in moving the entire body. Some of these patients can only move their eyes. In severe conditions of the progressive motor neuron diseases, patients cannot move their eyes nor can they speak. Establishing an efficient communication channel without overt speaking and hand motions makes the patient's life a bit easier and increases their quality of life.

ALS occurs in between 4 and 8 out of every 100,000 individuals and only a small percentage of cases arise from a known genetic cause (Parker \& Parker, 2007). Concerning other motor neuron diseases or speaking and muscular disabilities, there are more than 100 million potential users in need of alternative channels such as brain computer interface (BCI) for communicating with their environment or for controlling devices (Guger, 2008). Considering life span extension and increasing causes of injuries including traffic accidents and explosions, which may result in spinal cord injuries in serious cases, the need for an efficient communication or control channel has been drastically increasing.

HCIs are a research field which includes interactions such as communication and device/machine control between a user and a computer. The aim of the HCI is to improve performance of the interaction, meaning a minimization of the barrier between the human and the computer. Accurate and fast interpretation of what the user wants to do as well as a correct understanding by the computer of the user's intentions or demand is the aim of this research field.

Man-machine interface (MMI), brain-machine interface (BMI) and BCI can be thought of as applications of HCIs. If communication or control is established directly from the brain, it is called BCI and it is the only method of interaction for the individuals with complete 
paralysis. Because these research fields are new, there is a need for development in terms of efficiency; meaning accuracy, reliability and quick responses are necessary. Many research groups from all over the world are focusing on HCI applications in order to improve alternative communication channels for the disabled. An efficient alternative channel for communication and control device without overt speaking and muscular movements is important to make life easier for individuals who are suffering from ALS or other illnesses that prevent proper limb and muscular responses. Because of this, the area of study related with HCIs has high expectations and are important for improving quality of life.

In this chapter, ALS related HCI in particular, is discussed. The very common field electroencephalogram (EEG) based BCI and other approaches in this field are presented. With interdisciplinary studies, developing new interfaces and interaction techniques are opening new research fields for investigation. Especially for the paralysis patients, the classical communication or control ways, such as overt speaking or hand motions cannot be used. Using bio-signals such as EEG and its various methodologies (i.e. P300, slow cortical potentials, etc), electrooculogram (EOG), hearth rate (HR) or galvanic skin response (GSR) as well as the hemoglobin level which is related to oxygenation, are the only ways to send messages or control signals to devices regarding user's demands, intentions or expectations. This does not only give the patients the potential ability to give messages on computer screens and control a powered wheelchair or robot arm without muscular movements, but also can be potentially useful for the elderly as well.

The aim of this chapter is to present the state of the art of the technology on HCIs. This chapter also addresses the use of different bio-signals individually or the integrated hybrid/integrated multi-modal system approach for communication and control with high performance. In order to increase performance, processing combined bio-signals and multi-modal integrated systems will be discussed. For this purpose, several bio-signals such as EEG, EOG, and functional near infrared (fNIR) spectroscopy based system research are introduced.

\section{Human computer interactions}

In general, HCIs are related to the adaptation of a human and a computer. HMIs adapt human demands to the machine. Human computer interface operation requires the effective interaction of two sides; user and system. Here, a system can be an integrated system of computers and a word speller, a robot arm, a powered wheelchair, house appliances such as door locks, TVs or musical instruments, etc. In order to reflect intent/demands, instead of overt speaking or muscular movements, other bio-signals can be used. Effective BCIs may serve as assessment tools and adaptive systems for HCI for able-bodied people and be proven for people with severe motor neuron diseases.

Research in this field is typically focused on several areas of improvement for HCIs in order to increase its usefulness and effectiveness. These areas are:

i. High performance
a. Accuracy
b. Reliability
c. Fast
d. Robustness

ii. User friendliness (including user training)

iii. Ease of application

iv. Cost effectiveness. 
In short, HCI should reflect user demands and expectations accurately and quickly. The next sections of this chapter will introduce the EEG, EOG, NIRS based systems, as they are technologies that show much promise. In addition to these technologies, electrocorticography (ECoG), functional magnetic resonance imaging (fMRI), galvanic skin response (GSR) and heart rate (HR) based systems; and multi-modal integrated design rationale are introduced briefly.

\subsection{Brain computer interfaces}

From a broad perspective, BMI refers to the interface between a brain and a machine (for review Lebedev \& Nicolelis, 2006). In this section, the common term - brain computer interface (BCI) will be presented. BCI can be described as a translation of human intentions into a control signal without using the muscles. The aim of $\mathrm{BCI}$ is to provide communication and control for people with severe motor disabilities.

The BCI system translates the signals that are encoded by the user's intentions into messages and control commands. Research in this field has been rapidly growing in neuroscience and bioengineering. Specifically, this technology is promising for users with motor neuron diseases. Table 1 shows the estimated potential users of BCI. According to this table, there are more than 100 million potential users in the world.

\begin{tabular}{|l|l|}
\hline Type of the Disease & Number of Patients \\
\hline Amyotrophic Lateral Sclerosis & $400,000 / 3,000,000$ \\
\hline Multiple Sclerosis & $2,000,000$ \\
\hline Muscular Dystrophy & $1,000,000$ \\
\hline Brainstem Stroke & $10,000,000$ \\
\hline Cerebral Palsy & $16,000,000$ \\
\hline Spinal Cord Injury & $5,000,000$ \\
\hline Postpolio Syndrome & $7,000,000$ \\
\hline Guillain-Barre Syndrome & 70,000 \\
\hline Other types of Stroke & $60,000,000$ \\
\hline
\end{tabular}

Table 1. Potential users of BCI in the world (Guger, 2008).

Electrical brain activities (electroencephalography, EEG) related to human intentions can be monitored using electrodes attached to the scalp surface, non-invasively. EEG signals are gross potential of the thousands of neurons, roughly reflecting bodily functions. Because the skull and scalp play the role of a barrier for the electrical signals, EEG signals have low amplitudes (in micro-volts scale) and exist in the $0.5-30 \mathrm{~Hz}$ frequency band. Figure 1 shows the ongoing time series EEG signal and its power spectral density. As it is shown from this figure, $8-12 \mathrm{~Hz}(\alpha$ band) and $26 \mathrm{~Hz}$ ( $\beta$ band) components are dominant.

In order to increase efficiency, brain electrical signals can be recorded by subdural electrodes (electrocorticogram, ECoG), invasively. ECoGs are neuronal activity that is acquired from smaller cortical areas when compared to EEGs. Epidural or subdural recording is less invasive than intra-cortical recording. While their applications are difficult, the resolution of these recordings can be significantly higher than conventional EEG. The BCI usefulness of intra-cortical signals is promising (Wolpaw, 2003). 


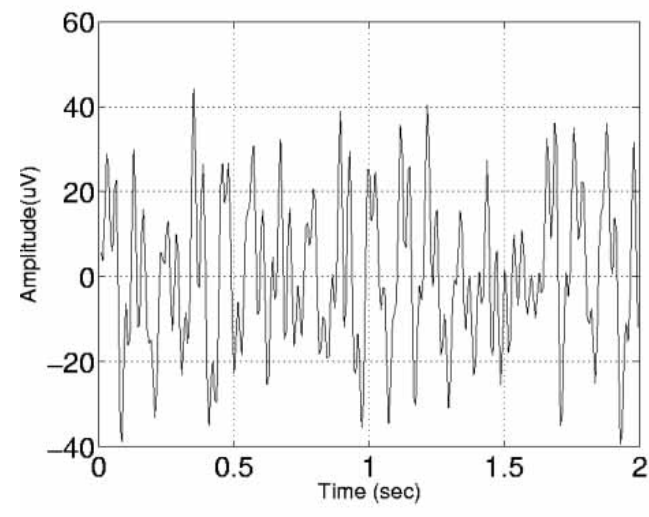

(a)

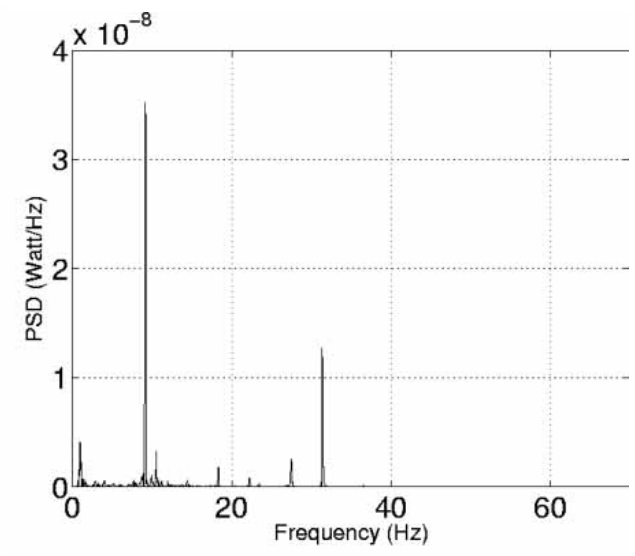

(b)

Fig. 1. Ongoing EEG recording from 27-year old male subject's occipital lobe: (a) the time series signal, and (b) power spectral density.

BCI systems can provide a communication and control channel, which bypasses conventional neuromuscular pathways involved in speaking or movement activity made to manipulate objects (Wolpaw et al., 2002). The command for device control can be generated by self-regulated $\mu$ rhythms (Wolpaw et al., 2000), motor imagery (Pfurtscheller et al., 2000) or a visual evoked potential (Sutter, 1992).

An EEG based BCI system can use the signals listed below.

- $\quad$ P300 response,

- $\quad$ steady state visual evoked potential (SSVEP),

- event related desynchronisation (ERD),

- $\quad$ slow cortical potential (SCP) and

- $\quad$ sensorimotor rhythm (SMR).

P300 and SSVEP methods require external stimulation that the user has to focus attention on or gaze into flickering lights, which the other methods do not need (self-paced systems). Because of this, the user is not free to decide on performing an action; the user depends on computer software for the synchronization. Each of these methods is advantageous or disadvantageous with respect to performance, information transfer rate and user training time. The BCI systems may not use just one of these approaches, but may use a combination of two or more signals as a hybrid approached system.

As an event related potential (ERP), the common method is using the P300 response (Farwell \& Donchin, 1988). The P300 response is elicited between $300 \mathrm{msec}$ and $400 \mathrm{msec}$ after stimulation, as it is shown in figure 2. Because ERPs are so small, for clarity, signal (epoch) averaging is necessary. The latency and amplitude may change from user to user, however the shape of the signal is roughly the same. In addition to using P300 response, SSVEPs (Gao et al, 2003) and SCPs, $\mu(8-12 \mathrm{~Hz})$ and high $\beta(18-26 \mathrm{~Hz})$ rhythms (sensorimotor applications) are used in BCI applications.

The SSVEP is a brain response evoked mainly in the visual and parietal cortices as a response to flickering visual stimuli. The SSVEP has gained popularity in the BCI research because it provides advantages in terms of speed and robustness. ERD and Event-related synchronization 
was quantified by the most reactive frequency bands were chosen then band-pass filtered within those bands. SCPs are slow shifts of the EEG with duration from $300 \mathrm{msec}$ to several seconds. The group of SCPs includes the contingent negative variation, premovement potentials, the Bereitschaftspotential and expectancy waves (Pfurtscheller et al., 2005).

While these approaches are important for the detection of imagery motor activities, the approach of the P300 waveform is very common in BCI applications. As it is shown figure 2, the P300 response is a large, positive potential (conventionally opposite polarization) that has been studied extensively within the context of the oddball paradigm. Donchin and colleagues first reported the use of the P300 for BCI communication (Donchin et al., 2000). International approaches for the detection of P300 speller, a matrix of grey symbols on a dark background (virtual keyboard) are shown in figure 3.

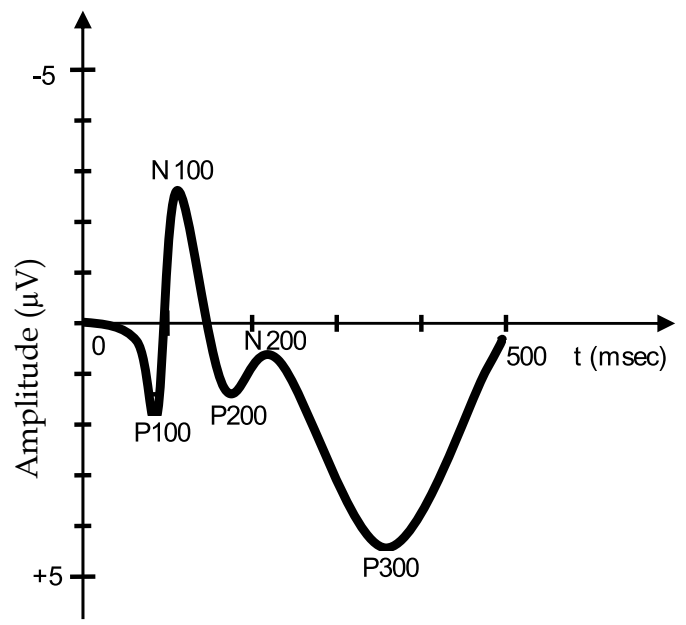

Fig. 2. Typical averaged event related potentials after stimulation. In BCI applications P300 component is extensively used.

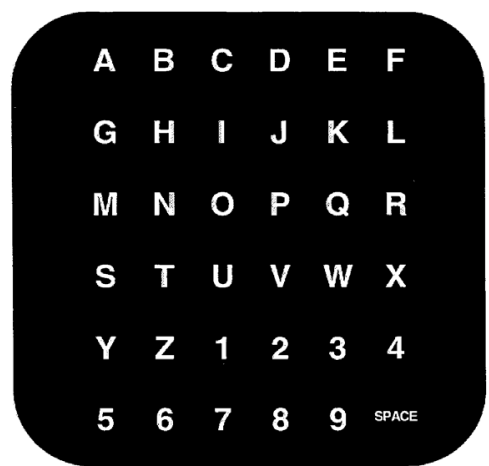

Fig. 3. The matrix for P300 speller. The stimulus matrix monitored by the subject. Typically, one of the rows or columns of the matrix was intensified every $125 \mathrm{~ms}$ (The matrix is proposed first by Donchin and colleagues 1988). 
The P300 speller paradigm has been common as a promising communication tool. Using the P300, a virtual keyboard without using or requiring any activation of skeletal muscles can be realized (Farwell \& Donchin, 1988; Donchin et al., 2000; Sellers \& Donchin, 2006). In the virtual keyboard, rows and columns of the matrix were randomly intensified typically every 125 ms. A P300 was produced after attended character is flashed. The attended symbol was selected by averaging responses for rows and columns. Accurate performance is obtained in users with and without motor impairments. Users with ALS are able to use the P300 based, single-stimulus system using either auditory or visual presentations. The Oddball paradigm to implement using the commands: Yes, No, Pass, and End with three presentation modes: auditory, visual, and both auditory and visual were used (Sellers et al., 2006). Detecting P300 signals the mind spelled characters (words, sentences, stories), enable the communication-disabled via internet (van Kokswijk \& van Hulle, 2010) were realized.

People can learn to control EEG features consisting of SMR amplitudes and can use this control to move a cursor to a target on a screen. EEG recordings during right and left motor imagery allow users to establish a new communication channel. Such an EEG-based BCI can be used to develop a simple binary response for the control of a device (Guger et al., 2000). To control cursor movement $8-12 \mathrm{~Hz}(\mu)$ or $18-26 \mathrm{~Hz}(\beta)$ frequency bands over sensorimotor cortex can be used (Fabiani et al., 2004). In the standard one-dimensional application, the cursor moves horizontally from left to right at a fixed rate while vertical cursor movement is continuously controlled by SMR ( $\mu$ and $\beta$ rhythms) amplitude over left and/or right sensorimotor cortex (McFarland \& Wolpaw, 2005). Intention of movement of left or right index finger, or right foot is recognized in EEG signals (Peters et al., 2001).

The model usually employed in many BCI systems is presented in Figure 4. Here, messages can be word speller output and commands can be for the powered wheelchair, robot hand or domotic (house) appliances control commands.

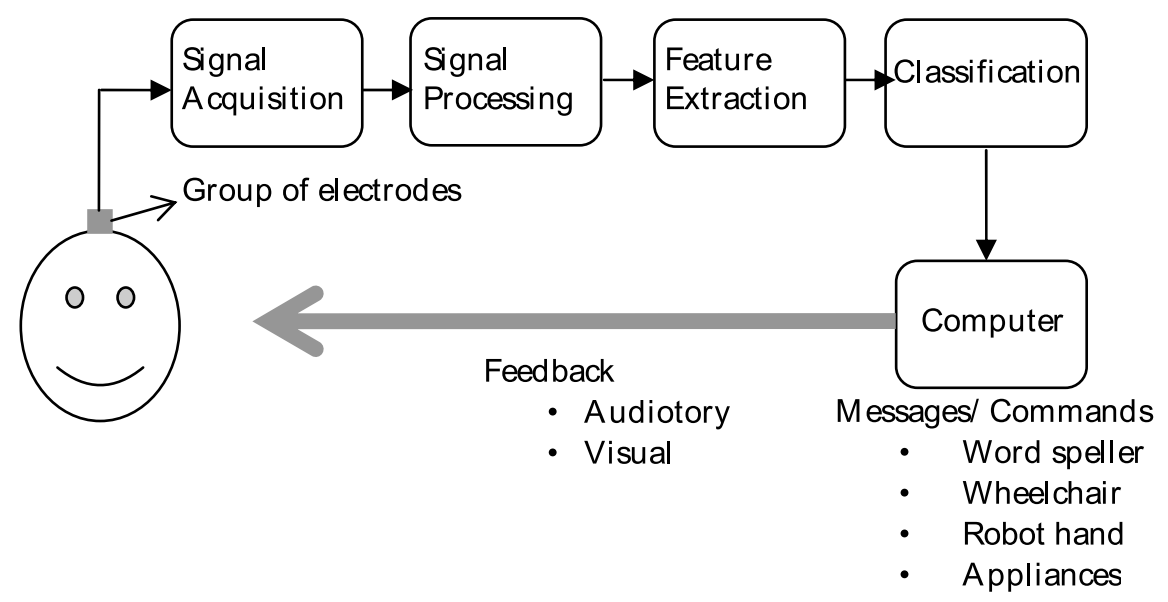

Fig. 4. A Typical BCI system.

To increase usefulness, BCI research groups are focusing several respects. In order to evaluate the usefulness of approaches for user intention, it is important to recognize that intention is normally formatted as a goal (Wolpaw, 2003). This goal may be "Write letter ' $A$ ' (On the screen)", "Move forward (powered wheelchair)" "Hit target (cursor)", "Grasp the 
glass", "Turn on the TV" or "Unlock the door", etc. After real time processing regarding the goal, the next step is goal-directed feedback generation.

The BCI systems may be self-paced. This means that the system allows communication or control whenever the user wishes. These systems' performance determined the true positive rate and the false positive rate. The true positive rate is the percentage of intentional control commands that are correctly detected by the BCI system. False positive rate are false positives generated by the system during the periods for which the user does not intend control.

A synchronous BCI uses external stimulation whereas in asynchronous BCI the subject makes self-paced decisions on when to switch from one mental task to the next. A virtual keyboard and a mobile robot which respond every $0.5 \mathrm{~s}$ were developed (Millan \& Mourino, 2003). Additionally, an asynchronous BCI analyzes and classifies EEG data continuously (Townsend et al., 2004). These systems are designed to process only one brain state in the ongoing EEG. For this purpose, only one channel may be sufficient. To control a powered wheelchair using a delayed response task a binary classification of left and right movement intentions were classified with a classification rate of over $80 \%$ from single trial EEG (Kaneswaran et al., 2010).

In order to improve the BCI system's performance, a rapid transition of various types of parameter estimation and classification algorithms to real-time implementation and testing (Guger, et al., 2001), classification accuracy and communication rate (Turnip et al., 2010) were realized. Identifying errors through the brain's reaction to mistakes is used to improve the robustness, flexibility, and reliability of BCIs (Buttfield et al., 2006). In addition to algorithmic improvement to increase performance, or in other words - high-resolution, multi-channel EEG systems have been developed to enhance the spatial information content of EEG activity. All these factors help to increasing the efficiency of BCI systems.

For standardization of BCI research, there is a documented general-purpose BCI research and development platform known as BCI2000. This platform can incorporate independent or a combination of any brain signals, signal processing methods, output devices and operating protocols (Wolpaw et al., 2003; Schalk, et al., 2004).

As a new approach to drive BCIs, functional magnetic resonance imaging (fMRI), have been used. The temporal resolution of the hemodynamic deoxyhemoglobin changes in the range of 1-2 seconds, while its spatial resolution is generally observable with the current imaging techniques at a few millimeters scale. Local hemodynamic response can be measured by fMRI. Hence, fMRI responses and cortical sources of EEG data are spatially related. It is possible to estimate the cortical activity with a spatial resolution of few millimeters and with a temporal resolution of milliseconds from noninvasive EEG measurements (Astolfi et al., 2010).

Although an fMRI based BCIs noninvasively records activity of the entire brain with a high spatial resolution, they are not suitable for everyday use. They have temporal delays of several seconds. However, they have good spatial resolution and they can sample the activity of deep brain structures (Lebedev \& Nicolelis, 2006). An fMRI-based BCI platform which performs data processing and feedback of the hemodynamic brain activity within 1.3 s (Weiskopf et al., 2004), psychophysiological markers (Nijboer et al., 2009), and communication using real-time fMRI (Eklund et al., 2010) were developed. In the later system, the subject in the MR scanner sees a virtual keyboard and steers a cursor to select different letters that can be combined to create words. The cursor is moved to the left by 
activating the left hand, to the right by activating the right hand, down by activating the left toes and up by activating the right toes (Eklund et al., 2010).

It can be concluded that as an alternative method for communication through speaking and muscular movements, BCIs are allowing communication and control for individuals with motor neuron disease such as ALS. A BCI system consists of recognition by a computation of the patterns of brain electrical activity on the scalp acquired from an array of electrodes. Although this technology is quite useful, it still needs to be developed in terms of efficiency.

\subsection{Electrooculography based systems}

Paralyzed patients are unable to communicate normally with their environment. For these patients, the only part of their body that is under their control, in terms of muscular movement, is their eyes. Some research in this area has been focused on investigating new efficient communication tools for paralyzed patients to translate their eye movements into appropriate communication messages or control signals.

With eye movements, a potential across the cornea and retina exists. This potential is called the cornea-retinal potential and it is the source of the electrooculogram (EOG). Communicating and controlling with EOG can be used for the disabled. An EOG based HCI device is able to recognize the subject's eye movements by using the electrical activity generated by the eye movements. EOG signals have certain patterns for each kind of eye movement (left, right, up, down, blink or wink). These signal patterns can be acquired and then recognized as signals which can be used for controlling external devices like a virtual keyboard, a powered wheelchair, a robot arm or a movable robot. As a very common application, the EOG-based virtual keyboard provides a means for paralyzed patients to write letters on a screen with eye movements without using a conventional keyboard. An EOG-based system for HCI application is presented in figure 5 and EOG signal samples are shown in figure 6.

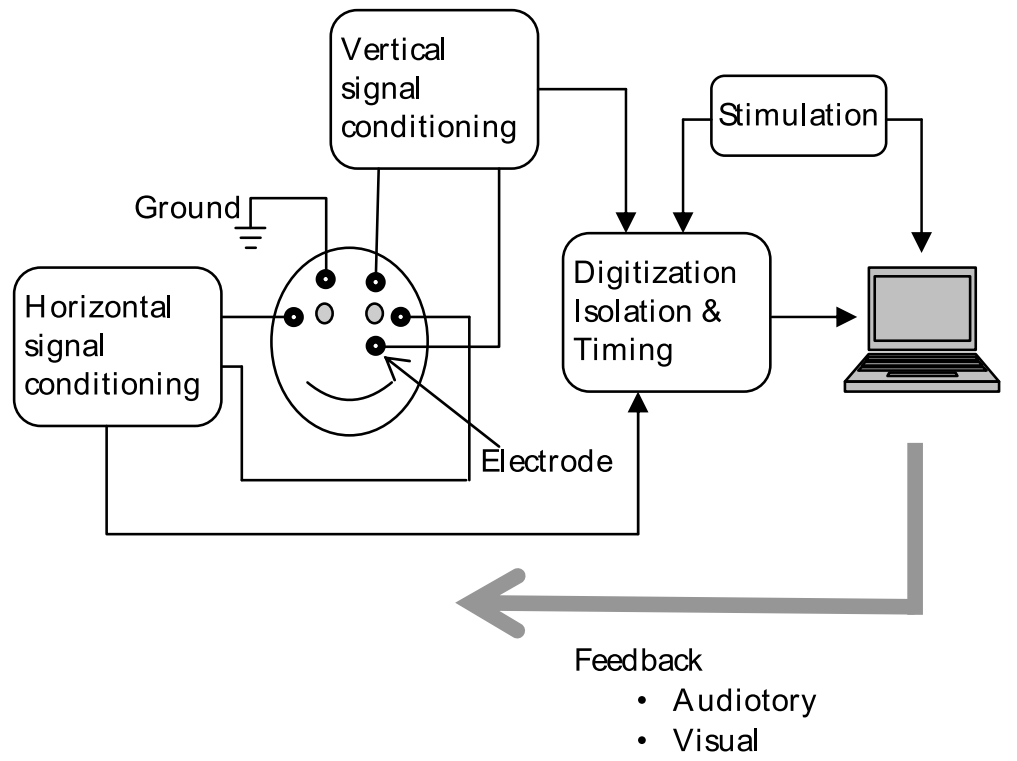

Fig. 5. Typical EOG-based interface. 


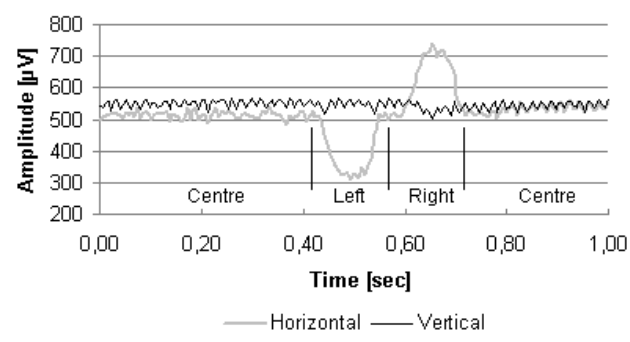

(a)

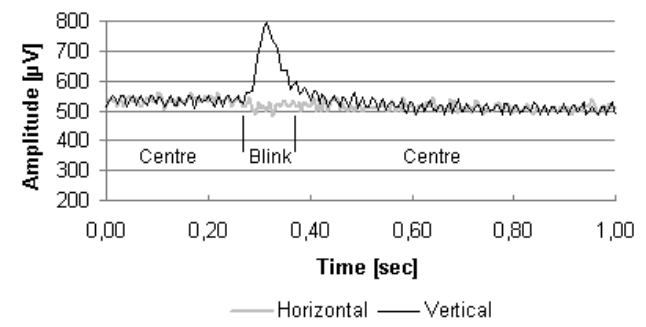

(c)

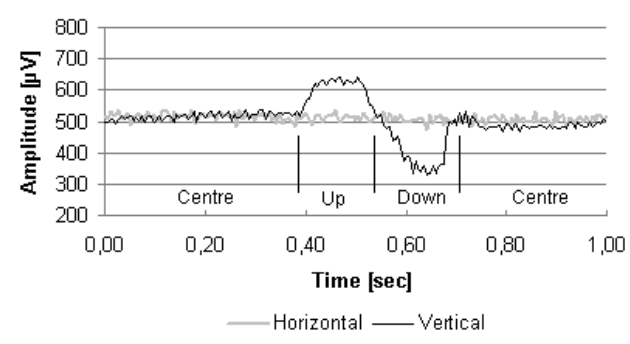

(b)

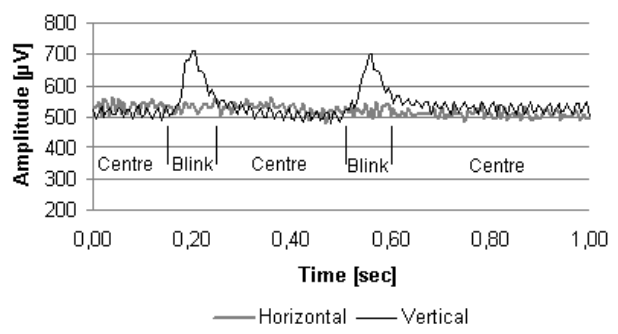

(d)

Fig. 6. Voluntary EOG signals: a) Horizontal, b) Vertical, c) Eye blink and d) double blink (Usakli \& Gurkan, 2010).

Many studies exist in the literature concerning the application of eye movements to the HCI (Bahill, 1982; Yoshiaki, 1997; Juhola, 1985; Allison et al., 1996; Hutchinson et al., 1989; Norris \& Wilson, 1997; Kuno, et al., 1998; Chen et al., 1999; Ihara et al., 2009). The research focused mainly on the word speller. Recognizing eye movements; such as left, right, up and down, and eye blinks to select characters from the virtual keyboard on the screen can use giving a message (Kherlopian et al., 2006; Akan \& Argunsah, 2007; Usakli et al, 2009). With the EOG based word speller, it is reported that 5 letter-word such as "Water" can be written in 25 seconds (Usakli \& Gurkan, 2010). The menus of this system are shown in figure 7. EOGcontrolled cursor interfaces, where the cursor can be controlled by eye movement (Tomita et al., 2006; Tamura et al., 2010) are realized, successfully.

As a communication device, a prototype of a head-mounted display with the eye-gaze detection function was developed which a user can operate by eye movements (Handa \& Ebisawa, 2008). With this device, the eye-gaze point was determined from the relative position between the pupil center and the corneal reflection of the light source which were detected by the camera. An eye-gaze controlled navigation and electromyography (EMG) enter (confirm) the selection of letter (Dhillon et al. 2009), and an eye-movement tracking system (Krueger \& Stieglitz, 2007; Deng et al., 2009; Septanto et al., 2009) were developed. Another promising EOG based method is a Morse code generator (Wu et al., 2007). Additionally, an EOG-based a powered wheelchair (Barea et al., 2002; Chung-Hsien et al., 2009) and a portable wireless device (Zheng et al., 2009) were realized. The EOG based device allows the patients to generate decisions on a screen by means of simple eye movement signals. These signals can be measured with EMG/EEG electrodes, without the need of complex systems or infra-red cameras. Then, patients are able to select letters on the 
screen or even communicate basic needs (food drinks, etc.) to the caregiver with a simple movement of their eyes (Usakli et al, 2009). All these studies show that EOG signals can be used as an input for efficient HCI applications. Since the EOG signals are larger, measurement of these signals are easy compared to EEG. This property makes EOG applications much more efficient.

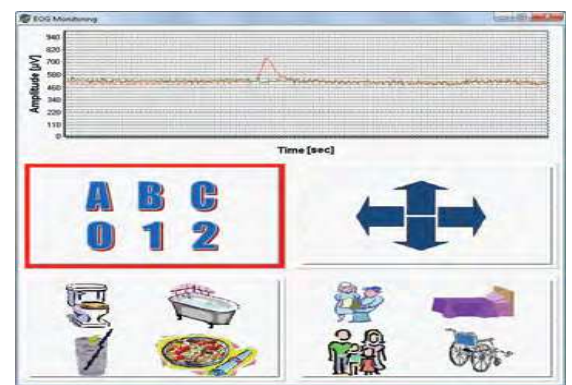

(a)

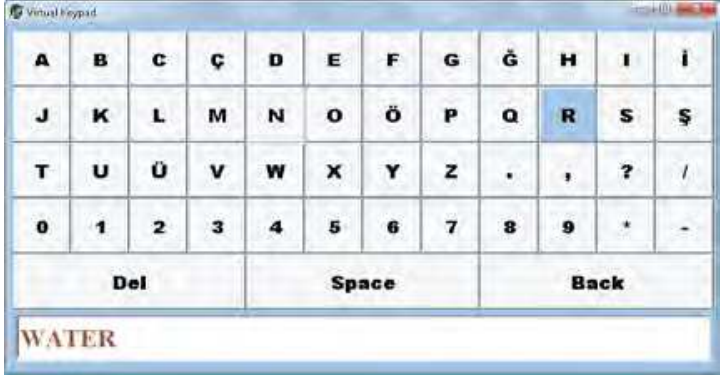

(b)

Fig. 7. The EOG-based user interface (Usakli \& Gurkan, 2010): a) Main menu, on the top, vertical and horizontal EOG signals are shown. b) Virtual keyboard: A 5-letter word can be written in 25 seconds.

\subsection{Near-infrared spectroscopy based systems}

Another approach for noninvasive BCI is based on optical means that measure brain activity by monitoring the hemodynamic response. Near Infrared Spectroscopy (NIRS) optical recording technology measures changes in the brain's oxygen absorption based on the optical properties of hemoglobin. Optical imaging spectroscopy can provide high spatial temporal resolution information about fractional changes in the hemodynamic response to increased neural activity (Mayhev et al, 2000). Human performance and cognitive activities such as attention, working memory, problem solving, etc., can be assessed by fNIR technology (Izzetoglu et al, 2007). NIRS technology usage is growing throughout the world for better understanding cortical activity during cognitive tasks.

NIRS is a spectroscopic method that uses about $800 \mathrm{~nm}$ wavelengths in the electromagnetic spectrum. The absorption spectrum in the near-infrared window is presented in figure 8. The level of light absorption related to the amount of oxyhemoglobin can be measured with detectors. While concentrating, brain uses much more oxygen than normal state. This demand meets with clean blood, then the number of oxyhemoglobin increases. Therefore, it causes more absorption of the light (Chance et al., 1998). This method gives an idea of oxygenation (changes of (de)oxy-hemoglobin) of blood in cortical capillary vessels.

The primary application of NIRS in the human body is seen through the measurement of the transmission and absorption of NIR light in human body tissues containing information about the changes in hemoglobin concentration. When a specific area of the brain is activated, the localized blood volume in that area changes quickly. Maximum response is observed between the 5th - 9th seconds (Malonek et al. 1997). Optical imaging can measure the location and activity of specific regions of the brain by continuously monitoring blood hemoglobin levels through the determination of optical absorption coefficients. 


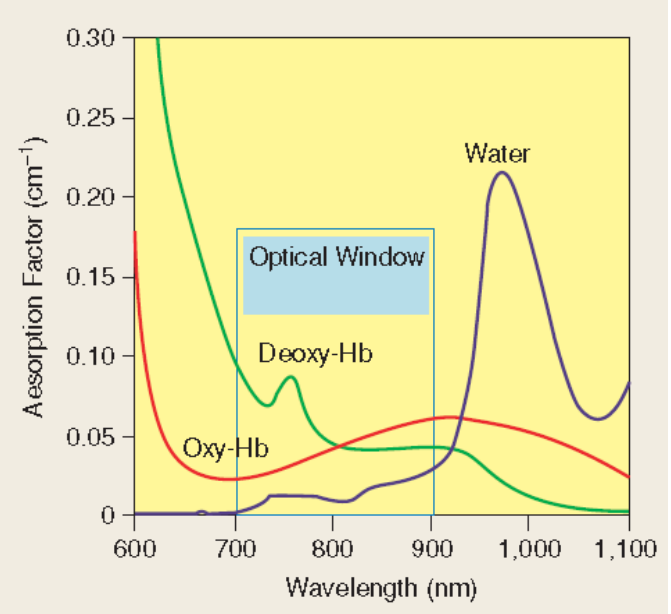

Fig. 8. Absorbtion spectrum in near-infrared window (Izzetoglu et al., 2007).

Figure 9 and 10 show measurement principles of oxygen level in a general NIR system. Photons transmitted with capillary vessels detected by detectors and measured photon intensity related to the oxygen level. fNIR spectroscopy can be used for BCI for the patients with ALS diseases (Bunce et al., 2006); to detect cognitive activity from prefrontal cortex elicited voluntarily (Ayaz et al., 2007).

\section{From control electronics}

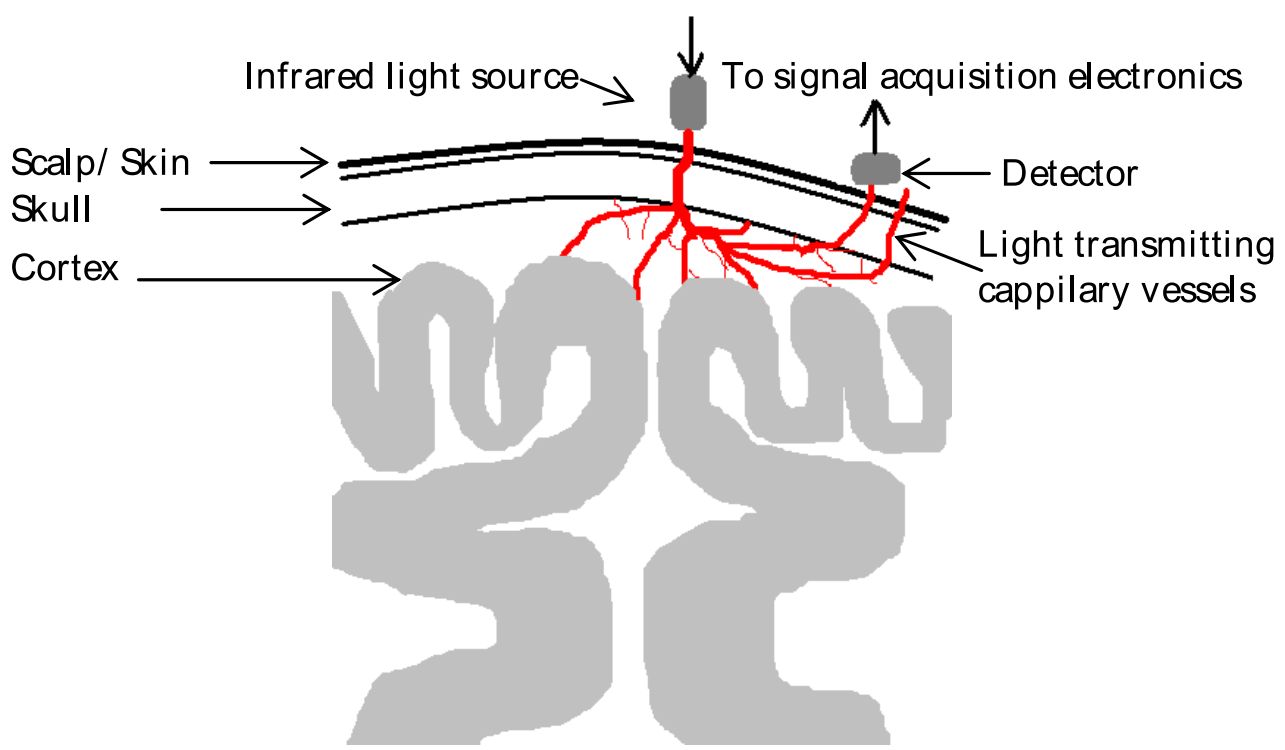

Fig. 9. Measurement of oxygen level in a NIR system. More light absorption means more hemoglobin, and consequently, more oxygenation. 


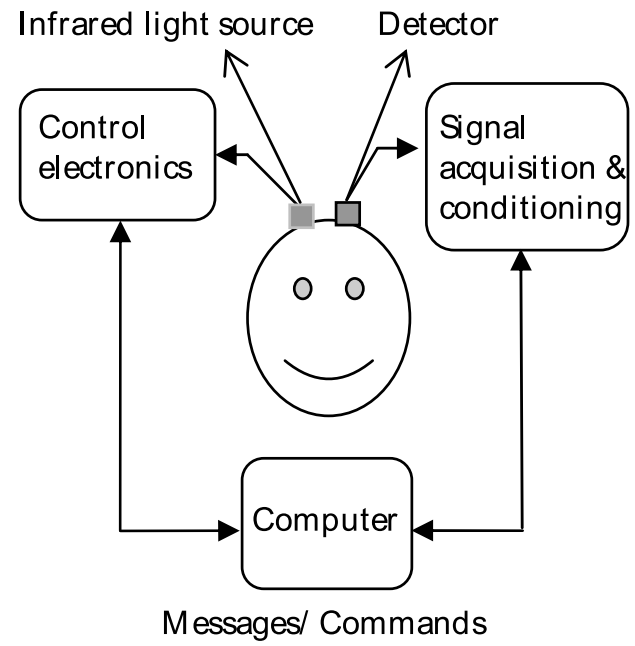

Fig. 10. The block diagram of a NIR system.

\subsection{Other approaches}

The behavior of active motor units identified via analysis of EMG signals recorded from the first dorsal interosseous muscle using a quadrifilar needle electrode is investigated. According to this study, the motor unit action potential waveforms recorded from patients were more complex than those recorded from control subjects as often observed in motor neuron diseases (Kasi et al., 2009). An eating assistant robot used to assist in eating independence was developed. This assistant robot is useful for people with severe disabilities. A spoon and a camera are attached on the tip of the robotic arm (Takahashi et al., 2001). Additionally, detecting the stress level of the computer user could possibly develop the computers' ability to respond intelligently and help calm negative emotional states of the user during HCI.

\section{Feature extraction and classification algorithms}

To increase the performance of the HCIs, algorithmic studies related to feature extraction and classification were realized. Motor imagery based BCI, the feature extraction, was performed with an adaptive autoregressive model and the classifier used was an adaptive quadratic discriminant analysis (Vidaurre et al., 2006). A new algorithm for single-trial online classification of imagery left and right hand movements was developed. This algorithm is based on time-frequency information derived from filtering EEG wideband raw data with causal Morlet wavelets, which are adapted to individual EEG spectra (Lemm et al., 2004). For motor imagery EEG, a new EEG recognition algorithm which combined the discrete wavelet transform with the backpropagation neural network was developed (MingAi et al., 2009). According to the results, performance of motor imagery based BCI using a single recording session of EEG or ECoG signals for each subject, is not sufficient. It was relatively easy to obtain classifiable signals quickly from most of the non-paralyzed subjects. However, it was proved that it is impossible to classify the signals obtained from the paralyzed patients by the same methods (Hill, et al., 2006). To detect the ERPs, EEG 
recordings are transformed into a Haar-wavelet series (Kawakami et al., 1996) and variational Kalman filtering (Sykacek et al., 2004) for adaptive classification in the BCI system was used. The later algorithm translates EEG segments adaptively into probabilities of cognitive states. It allows for nonstationarities in the joint process over cognitive states and generated EEG which may occur during a consecutive number of trials. The wavelet features are used to determine the characteristic of eye movement waveform (Daud \& Sudirman, 2010).

A new two-stage approach to extract the $\mu$ rhythm component was developed. The first stage uses second-order blind identification with stationary wavelet transform to automatically remove the artifacts. In the second stage, second-order blind identification is applied again to find the $\mu$ rhythm component. In this method artifact removal enhances the extraction of the $\mu$ rhythm component ( $\mathrm{Ng}$ \& Raveendran, 2009). For classification of motor execution signals, fractal approach provides promising results (Usakli, 2010). An EEG based BCI for users to control a cursor on a computer display is one of the common study area. The developed system uses an adaptive algorithm, based on kernel partial least squares classification, to associate patterns in multichannel EEG frequency spectra with cursor controls (Trejo et al., 2006). For the BCI related classification review can be found in (Lotte et al., 2007).

\section{Hybrid approach of human computer interaction}

To increase HCI efficiency, hybrid approaches offer good results. Three physiological signals: blood volume pulse, galvanic skin response and pupil diameter, to automatically monitor stress were used, successfully (Zhai et al., 2005). A vision-based multimodal human computer interface system using eye and hand motion tracking was developed. This vision-based virtual interface integrates the function of the motion tracking of eye blinking and hand gestures with the function of their recognition as a virtual interface (Shin \& Chun, 2007). EEG error-related signals present a hybrid approach for HCI. This approach uses human gestures to send commands to a computer and exploits brain activity to provide implicit feedback about the recognition of such commands (Chavarriaga et al., 2010).

\subsection{Progressing study for hybrid multi-modal human computer interaction}

In order to contribute to the HCI field, a novel multi-modal integrated design is completed and establishing an efficient communication and control channel for individuals with motor neuron diseases such as ALS has been continuing. The preliminary results are promising. Using experience in relevant fields such as EEG (Usakli \& Gencer, 2007a; Usakli \& Gencer, 2007b), EOG (Usakli \& Gurkan, 2010; Usakli et al., 2009), BCI prototyping (Erdogan et al, 2009), feature extraction (Usakli, 2010), this novel system attempts to increase usefulness and performance. HR and GSR signals are to be processed with the bio-signals mentioned above, simultaneously. Evaluation of these signals is whether individually or combined, depends on the user. The situation of the severity of the disease also determines the mode of the hybrid multi-mode operation. Through focusing on one of the tasks and sending correct or wrong messages, the bio signals of the subjects will be acquired and processed. Detecting these changes can be used to send data without speaking or control the device. 


\subsection{Design rationale}

The novel system is microcontroller based and battery powered. Data is transferred via optic fiber. To remove the dc level and $50-\mathrm{Hz}$ power line noise, differentiating approach is used. This approach is more successful and practical than the classical methods in the application. After signal conditioning; including filtering and amplification, the analog signal is digitized (at least 12 effective bits) at variable sampling rates and then transferred to the PC via optic fiber. To classify bio-signals, feature extraction with the fractal approach (Usakli, 2010) and wavelet transformed data is applied to artificial neural networks for classification. By using a user-friendly interface, the virtual keyboard and controlling pad allows messages to be given, and some other needs such as cleanup or medical assistance can be selected.

The integrated HCI system provides with high 1) efficiency, 2) usefulness, 3) robustness 4) accurate and reliable output, 5) fast response, 6) user friendly, 7) flexibility, 8) cheap, and 9) designed available components.

\section{Conclusions and discussions}

ALS is a progressive disease that affects the control of muscle movement by damaging motor neurons. ALS kills the pyramidal neurons of the motor cortex as a corollary muscular functions deteriorate rapidly. For the present, there is no known cure for ALS. Because of these movement and muscular disabilities, these patients need an efficient alternative channel to communicate with their environment or to control devices. In this chapter, HCIs especially BCI technology focused on ALS patients, are presented.

The EEG-based BCI systems represent the only technology for severely paralyzed patients to increase or maintain their communication and control needs. The P300 paradigm for the EEG-based BCI systems is due to the fact that such waveform occurs spontaneously for many of the subjects without need of particular training, which is be useful for increasing the quality of life for the patients. EEG is still the most attractive and popular technology for clinical BCI.

The EOG-based side of the system seems more accurate and fast when compared to the EEG-based systems. It must be noted that the solution for the EOG system is cheaper when compared to the EEG solution and can be used as a first step for the hybrid device for all users. The general idea of a hybrid device is to familiarize the patient with a unique interface, while the user can switch the bioelectric signal for the communication/control of the external devices.

NIRS is a non-invasive optical technique, suitable to assess functional activity by measuring cortical oxygenation $\left(\mathrm{HbO}_{2}\right)$ and deoxygenation $(\mathrm{Hb})$. This technology is also a promising and cheap technology for establishing efficient alternative channel, however needs more study on this field to prove ability of efficiency.

In order to increase the usefulness and improve performance, hybrid approaches and multimodal designs should be investigated. For EEG based BCI systems two or more signal of: P300 response, steady state visual evoked potential, event related desynchronisation, slow cortical potential, sensorimotor rhythms and error-related potentials can be used for an efficient hybrid system. There is no sufficient study in the literature concerning multi-modal system designs. Combination of several bio-signals such as: EEG, EOG, NIRS, HR and GSR, etc offer an improved performance results. The usage of this two or several signals combination may be used depends on the situation of the disability. 


\section{References}

Akan, B. \& Argunsah, A.O. (2007). A Human-Computer Interface (HCI) Based on Electrooculogram (EOG) for Handicapped, Signal Processing and Communications Applications SIU 2007 IEEE 15th, Eskisehir, Turkey, 11-13 June 2007.

Allison, R.S.; Eizenman, M. \& Cheung, B. (1996). Combined Head and Eye Tracking System for Dynamic Testing of the Vestibular System, IEEE Trans. on Biomedical Engineering, vol.43, no.11, pp.1073-1082.

Astolfi, L.; Gonzalez Andino, S.; De Vico Fallani, F. \& Babiloni, F. (2010). Processing of Brain Signals by Using Hemodynamic and Neuroelectromagnetic Modalities, Computational Intelligence and Neuroscience, 2010, ID 934180, pp.1-2.

Ayaz, H.; Izzetoglu, M.; Bunce, S.; Heiman-Patterson \& T. Onaral, B. (2007). Detecting Cognitive Activity Related Hemodynamic Signal for Brain Computer Interface Using Functional Near Infrared Spectroscopy, Neural Engineering, 2007. CNE'07. 3rd International IEEE/EMBS Conference on, 342, Kohala Coast, HI, USA, 2-5 May 2007.

Bahill, A.T. (1982). Prediction Final Eye Position Halfway Through a Saccade, IEEE Trans. on Biomedical Engineering, vol.30, no.12, pp.781-786.

Barea, R.; Boquete, L.; Mazo, M. \& Lopez, E. (2002). System for Assisted Mobility Using Eye Movements Based on Electrooculography, Neural Systems and Rehabilitation Engineering, IEEE Transactions on, Vol. 10 4, pp. 209 - 218, ISSN: 1534-4320

Bunce, S.; İzzetoglu, M.; İzzetoglu, K.; Onaral, B. \& Pourrezaei, K. (2006). Functional Near Infrared Spectroscopy: An Emerging Neuroimaging Modality, IEEE Engineering in Medicine and Biology Magazine, Special Issue on Clinical Neuroengineering, 25(4): pp. 54-62.

Buttfield, A.; Ferrez, P.W. \& Millan, Jd.R. (2006). Towards a Robust BCI: Error Potentials and Online Learning, Neural Systems and Rehabilitation Engineering, IEEE Transactions on, Vol. 14 2, pp. 164 - 168ISSN: 1534-4320

Chance, B.; Anday, E.; Nioka, S.; Zhou, S.; Hong, L.; Worden, .K.; Li, C.; Murray, T.; Ovetsky, Y.; Pidikiti, D. \& Thomas, R. (1998). A Novel Method for Fast Imaging of Brain Function, Non-invasively, With Light. Optics Express, 2, 10.

Chavarriaga, R.; Biasiucci, A.; Forster, K.; Roggen, D.; Troster, G. \& Millan Jdel, R. (2010). Adaptation of Hybrid Human-computer Interaction Systems Using EEG Errorrelated Potentials, Conference Proceedings IEEE Eng Med Biol Soc. pp. 4226-4229, Argentina, Aug. 31-Sept. 42010.

Chen, S.; Tsai T. \& Luo, C. (1999). Portable Clinical EOG Instrument System, Proc. of the First Joint BMES/EMBS Conference, pp.858-859, Atlanta, USA, 13-16 Oct. 1999.

Chung-Hsien, K.; Yi-Chang, C.; Hung-Chyun, C. \& Jia-Wun S. (2009). Eyeglasses based electrooculography human-wheelchair interface Systems, Man and Cybernetics, 2009. SMC 2009. IEEE International Conference on, San Antonio, TX, USA, pp. 4746 4751, ISSN: 1062-922X, 11-14 Oct. 2009.

Daud, W.M.B.W. \& Sudirman, R. (2010). A Wavelet Approach on Energy Distribution of Eye Movement Potential Towards Direction, Industrial Electronics \& Applications (ISIEA), 2010 IEEE Symposium on, Penang, Malaysia, 3-5 Oct. 2010.

Deng, L.Y.; Chun-Liang Hsu Tzu-Ching Lin Jui-Sen Tuan Yung-Hui Chen, (2009). EOGBased Signal Detection and Verification for HCI, Machine Learning and Cybernetics, 2009 International Conference on, 12-15 July 2009, Vol. 6, 3342, Baoding, Hebei, China, ISBN: 978-1-4244-3702-3, 2009 
Dhillon, H.S.; Singla, R.; Rekhi, N.S. \& Jha, R. (2009). EOG and EMG Based Virtual Keyboard: A Brain-Computer Interface, Computer Science and Information Technology, ICCSIT 2009. 2nd IEEE International Conference on, 8-11 Aug. 2009, Beijing, China.

Donchin, E.; Spencer, K.M. \& Wijesinghe, R. (2000). The Mental Prosthesis: Assessing the Speed of a P300-Based Brain-Computer Interface, IEEE Transactions on Rehabilitation Engineering, Vol. 8, No. 2, pp. 174-178.

Eklund, A.; Andersson, M.; Ohlsson, H.; Ynnerman, A. \& Knutsson, H. (2010). A Brain Computer Interface for Communication Using Real-Time fMRI, Pattern Recognition (ICPR), 2010 20th International Conference on, Istanbul, Turkey, pp. 3665, ISSN : 1051-4651, 23-26 Aug. 2010.

Erdoğan, B.; Akıncı, B.; Acar, E.; Uşaklı, A. B. \& Gençer, N. G. (2009). Prototype Hardware Design for Brain Computer Interface Applications, 14. Biyomedikal Mühendisliği Ulusal Toplantısl, Izmir, Turkey, 20-22 Mayis 2009.

Fabiani, G.E.; McFarland, D.J.; Wolpaw, J.R. \& Pfurtscheller, G. (2004). Conversion of EEG Activity into Cursor Movement by a Brain-Computer Interface (BCI), Neural Systems and Rehabilitation Engineering, IEEE Transactions on, Vol. 12 3, pp. 331 - 338, ISSN: 1534-4320.

Farwell L.A. \& Donchin, E. (1988). Talking off the Top of Your Head: A Mental Prosthesis Utilizing Event-Related Brain Potentials, Electroencephalogr.Clin. Neurophysiol., Vol. 70, pp. 510-523.

Gao, X.; Xu, D.; Cheng, M. \& Gao, S. (2003). A BCI-Based Environmental Controller for the Motion-Disabled, Neural Systems and Rehabilitation Engineering, IEEE Transactions on, Vol. 11, 2, pp. 137 - 140, ISSN: 1534-4320

Gevins, A.; Brickett, P.; Costales, B.; Le, J. \& Reutter, B. (1990). Beyond Topographic Mapping: Towards Functional-Anatomical Imaging with 124-Channel EEGs and 3D MRIs, Brain Topogr. 3, pp. 53-64.

Guger C. (2008). Brain Computer Interface, Lecture notes in The 4th International Summer School on Emerging Technologies in Biomedicine: Advanced Methods for the Estimation of Human Brain Activity and Connectivity, Applications to Rehabilitation Engineering, Patras, Greece, 29 June - 4 July 2008.

Guger, C. Schlogl, A. Neuper, C. Walterspacher, D. Strein, T. Pfurtscheller, G. (2001). Rapid Prototyping of an EEG-Based Brain-Computer Interface (BCI), Neural Systems and Rehabilitation Engineerin, g IEEE Transactions on, Vol. 9, 1, 49, ISSN : 1534-4320.

Guger, C.; Ramoser, H. \& Pfurtscheller, G. (2000). Real-Time EEG Analysis with SubjectSpecific Spatial Patterns for a Brain-Computer Interface (BCI), Rehabilitation Engineering, IEEE Transactions on, (Dec 2000), Vol. 84 , pp. 447 - 456, ISSN: 10636528

Handa, S. \& Ebisawa, Y. (2008). Development of Head-Mounted Display with Eye-Gaze Detection Function for the Severely Disabled, Virtual Environments, HumanComputer Interfaces and Measurement Systems, VECIMS 2008 IEEE Conference on, İstanbul, Turkey, 14-16 July 2008.

Hill, N.J.; Lal, T.N.; Schroder, M.; Hinterberger, T.; Wilhelm, B.; Nijboer, F.; Mochty, U.; Widman, G.; Elger, C.; Scholkopf, B.; Kubler, A. \& Birbaumer, N. (2006). Classifying EEG and ECoG Signals Without Subject Training for Fast BCI Implementation: Comparison of Nonparalyzed and Completely Paralyzed Subjects, Neural Systems and Rehabilitation Engineering, IEEE Transactions on, Vol. 14, 2183. 
Hutchinson, T.E.; White, K.P.; Martin, W.N.; Reichert K.C. \& Frey, L.A. (1989). HumanComputer Interaction Using Eye-Gaze Input, IEEE Trans. on Systems, Man and Cybernetics, vol.19, issue 6, pp.1527-1534.

Ihara, T.; Sugi, T.; Eriguchi, M.; Asami, T. \& Nakamura, M. (2009). Construction of Simple Communication Method by Use of Neuro-Biological Signals, ICCAS-SICE, 2009, pp. 3152, Fukuoka, Japan, 18-21 Aug. 2009.

Izzetoglu, M.; Bunce, S. C.; Izzetoglu, K.; Onaral, B. \& Pourrezaei, K. (2007). Functional Brain Imaging, Using Near-Infrared Technology Assessing Cognitive Activity in RealLife Situations, IEEE Engineering in Medicine and Biology Magazine, pp. 38-46.

Juhola, M. (1985). Detection of Saccadic Eye Movements Using a Non-Recursive Adaptive Digital Filter, Computer Methods and Programs in Biomedicine, vol.21, pp.81-88.

Kaneswaran, K.; Arshak, K.; Burke, E. \& Condron, J. (2010). Towards a Brain Controlled Assistive Technology for Powered Mobility, Engineering in Medicine and Biology Society (EMBC), 2010 Annual International Conference of the IEEE, Aug. 31 2010-Sept. 4 2010, Buenos Aires, Argentina, 4176, ISSN : 1557-170X

Kasi, P.K.; Krivickas, L.S.; Meister, M.; Chew, E.; Bonato, P. ; Schmid, M.; Kamen, G. \& Pu Liu Clancy, E.A. (2009). Characterization of Motor Unit Behavior in Patients with Amyotrophic Lateral Sclerosis, Conference on Neural Engineering NER'09 4th International IEEE/EMBS, Antalya, Turkey, 2009.

Kawakami, T.; Inoue, M.; Kobayashi, Y. \& Nakashima, K. (1996). Application of Event Related Brain Potentials to Communication Aids, Engineering in Medicine and Biology Society, 1996. Bridging Disciplines for Biomedicine, Proceedings of the 18th Annual International Conference of the IEEE, Vol.: 5, 2229, Amsterdam, Holland, 31 Oct -03 Nov 1996.

Kherlopian, A.R.; Gerrein, J.P.; Yue, M.; Kim, K.E.; Kim, J.W.; Sukumaran, M. \& Sajda, P. (2006). Electrooculogram Based System for Computer Control Using a Multiple Feature Classification Model, Engineering in Medicine and Biology Society, EMBS'06. 28th Annual International Conference of the IEEE, New York, USA, 2006.

Kuno, Y.; Yagi, T. \& Uchikawa, Y. (1998). Development of Eye Pointer with Free HeadMotion, Proc. of the 20th Annual International Conference of the IEEE Engineering in Medicine and Biology Society, Vol. 20, No 4, pp. 1750-1752.

Lebedev, M. A. \& Nicolelis, M. A. L. (2006). Brain-machine Interfaces: Past, Present and Future, Trends in Neurosciences, Vol. 29 No.9, pp. 536-546.

Lemm, S.; Schafer, C. \& Curio, G. (2004). BCI competition 2003-Data Set III: Probabilistic Modeling of Sensorimotor $\mu$ Rhythms for Classification of Imaginary Hand Movements, Biomedical Engineering, IEEE Transactions o, $n$ Vol. 51 6, pp. 1077 - 1080, ISSN: 0018-9294.

Li, M. A.; Wang, R.; Hao, D. M. \& Yang, J.F. (2009). Feature Extraction and Classification of Mental EEG for Motor Imagery, Natural Computation, 2009 ICNC '09 Fifth International Conference on, Vol. 2, pp. 139., Tianjin, China, 14-16 Aug. 2009.

Lotte, F.; Congedo, M.; Lécuyer,; A. Lamarche, F. \& Arnaldi, B. (2007). A Review of Classification Algorithms for EEG-based Brain-Computer Interfaces, Journal of Neural Engineering, Vol.: 4, 2, pp. 1-24, ISSN: 17412560.

Malonek, D.; Dirnagl, U.; Lindauer, U.; Yamada, K.; Kanno, I. \& Grinvald, A. (1997). Vascular Imprints of Neuronal Activity: Relationships Between the Dynamics of Cortical Blood Flow, Oxygenation and Volume Changes Following Sensory Stimulation, Proc. Natl. Acad. Sci. Vol. 94, pp 14826-14831, USA, December 1997. 
Mayhew, J.; Johnston, D.; Berwick, J.; Jones, M.; Coffey, P. \& Zheng, Y. (2000). Spectroscopic Analysis of Neural Activity in Brain: Increased Oxygen Consumption Following Activation of Barrel Cortex, NeuroImage, 12, pp. 664-675.

McFarland, D.J. \& Wolpaw, J.R. (2005). Sensorimotor Rhythm-Based Brain-Computer Interface (BCI): Feature Selection by Regression Improves Performance, Neural Systems and Rehabilitation Engineering, IEEE Transactions on, (Sept. 2005), Vol. 13 , pp. 372 - 379, ISSN: 1534-4320

Millan, J.R.; \& Mourino, J. (2003). Asynchronous BCI and Local Neural Classifiers: An Overview of the Adaptive Brain Interface Project, Neural Systems and Rehabilitation Engineering, IEEE Transactions on, (June 2003), Vol. 11 2, pp. 159 - 161

$\mathrm{Ng}$, S.C. \& Raveendran, P. (2009). Enhanced Rhythm Extraction Using Blind Source Separation and Wavelet Transform, Biomedical Engineering, IEEE Transactions on, Vol. 56,8 , pp. 2024.

Nijboer, F.; Carmien, S.P. ; Leon, E.; Morin, F.O.; Koene, R.A. \& Hoffmann, U. (2009). Affective Brain-Computer Interfaces: Psychophysiological Markers of Emotion in Healthy Persons and in Persons with Amyotrophic Lateral Sclerosis, Affective Computing and Intelligent Interaction and Workshops, ACII 2009. 3rd International Conference on, Amsterdam, Holland, 10-12 September 2009

Norris, G. \& Wilson, E. (1997). The Eye Mouse, an Eye Communication Device, IEEE Proc. of Bioengineering Conference, pp.66-67, Durham, N. Carolina , USA, 21-22 May 1997.

Nunez, P. L. (1995). Neocortical Dynamics and Human EEG Rhythms, Oxford University Press: New York, 1995, pp. 200-250.

Parker, J.N. \& Parker, P.M. (2007). Amyotrophic Lateral Sclerosis: A Bibliography and Dictionary for Physicians, Patients, and Genome Researchers, ICON Health Publications, USA, ISBN: 0-497-11327-9, pp. 4.

Peters, B.O.; Pfurtscheller, G. \& Flyvbjerg, H. (2001). Automatic Differentiation of Multichannel EEG Signals, (Jan. 2001), Vol. 48 1, pp. 111, ISSN : 0018-9294.

Pfurtscheller, G.; Neuper, C.; Guger, C.; Harkam, W.; Ramoser, H.; Schlögl, A.; Obermaier, B. \& Pregenzer, M. (2000). Current Trends in Graz Brain-Computer Interface (BCI) Research, IEEE Trans. Rehab. Eng., vol. 8, pp. 216-219.

Pfurtscheller, G.; Neuper, C. \& Birbaumer, N. (2005). Human Brain-Computer Interface, Motor Cortex In Voluntary Movements, A Distributed System For Distributed Functions, Alexa Riehle and Eilon Vaadia , (Ed), CRC Press, USA, Chapter 14, pp.1-35.

Schalk, G.; McFarland, D.J.; Hinterberger, T.; Birbaumer, N.; Wolpaw, J.R. (2004). BCI2000: a General-Purpose Brain-Computer Interface (BCI) System, Biomedical Engineering, IEEE Transactions on (June 2004), Vol.: 51 6, pp. 1034 - 1043, ISSN: 00189294.

Sellers, E. W. \& Donchin, E. (2006). A P300-Based Brain-Computer Interface: Initial Tests by ALS Patients, Clinical Neurophysiology, 117, pp.538-548.

Sellers, E.W.; Kubler, A. \& Donchin, E. (2006). Brain-Computer Interface Research at the University of South Florida Cognitive Psychophysiology Laboratory: The P300 Speller. Neural Systems and Rehabilitation Engineering, IEEE Transactions on, Vol.14, No.2, (June 2006), ISSN : 1534-4320.

Septanto, H.; Prihatmanto, A.S.; Indrayanto, A. (2009). A Computer Cursor Controlled by Eye Movements and Voluntary Eye Winks Using a Single Channel EOG, Electrical Engineering and Informatics, Selangor, 2009. ICEEI'09. International Conference on, Issue Date: 5-7 Aug. 2009, pp. 117 - 120. 
Shin, G. \& Chun J. (2007). Vision-Based Multimodal Human Computer Interface Based on Parallel Tracking of Eye and Hand Motion, Convergence Information Technology International Conference on, 21-23 Nov. 2007, pp. 2443, Gyeongju, S. Korea.

Sutter, E. E. (1992). The Brain Response Interface: Communication Through Visuallyinduced Electrical Brain Response, J. Microcomput. Appl., vol. 15, pp. 31-45.

Sykacek, P.; Roberts, S.J. \& Stokes, M. (2004). Adaptive BCI based on Variational Bayesian Kalman Filtering: an Empirical Evaluation, Biomedical Engineering, IEEE Transactions on, (May 2004), Vol. 51 5, pp. 719 - 727, ISSN: 0018-9294.

Takahashi, Y.; Hasegawa, N.; Takahashi, K. \& Hatakeyama, T. (2001). Human Interface Using PC Display with Head Pointing Device for Eating Assist Robot and Emotional Evaluation by GSR Sensor, Robotics and Automation, 2001, Proceedings 2001 ICRA IEEE International Conference on, Vol. 4, pp. 3674, ISSN:1050-4729, Seoul, S. Korea, May 21-26 2001.

Tamura, H.; Miyashita, M.; Tanno, K. \& Fuse, Y. (2010). Mouse Cursor Control System Using Electrooculogram Signals, World Automation Congress WAC 2010, ISSN : 2154-4824, Kobe, Japan, 19-23 Sept. 2010.

Tomita, Y.; Igarashi, Y.; Honda, S. \& Matsuo, N. (1996). Electro-Oculography Mouse for Amyotrophic Lateral Sclerosis Patients, Bridging Engineering in Medicine and Biology Society Disciplines for Biomedicine Proceedings of the 18th Annual International Conference of the IEEE, Vol. 5, pp. 1780 - 1781, Amsterdam, Holland, 31 Oct-3 Nov 1996.

Townsend, G.; Graimann, B. \& Pfurtscheller, G. (2004). Continuous EEG Classification During Motor Imagery-Simulation of an Asynchronous BCI, Neural Systems and Rehabilitation Engineering, IEEE Transactions on, Vol. 12 2, pp. 258 - 265, ISSN: 15344320.

Trejo, L.J.; Rosipal, R. \& Matthews, B. (2006). Brain-Computer Interfaces for 1-D and 2-D Cursor Control: Designs Using Volitional Control of the EEG Spectrum or Steadystate Visual Evoked Potentials, Neural Systems and Rehabilitation Engineering, IEEE Transactions on, Vol. 14 2, pp. 225, ISSN : 1534-4320.

Turnip, A.; Hong, K.S.; Ge, S.S. \& Jeong, M. Y. (2010). Neural Networks Training Based on Sequential Extended Kalman Filtering for Single Trial EEG Classification, Knowledge and Systems Engineering KSE 2010 Second International Conference on, p. 85, Hanoi, Vietnam, 7-9 October 2010.

Usakli, A. B.; Gurkan, S.; Aloise, F.; Vecchiato, G. \& Babiloni, F.(2009). A Hybrid Platform Based on EOG and EEG Signals to Restore Communication for Patients Afflicted with Progressive Motor Neuron Diseases, 31st Annual International Conference of the IEEE EMBS, pp.543-546, Minneapolis, Minnesota, USA, September 2-6, 2009.

Usakli, A.B. \& Gencer N.G., (2007). A USB-Based 256-Channel Electroencephalographic Data-Acquisition System for Electrical Source Imaging of the Human Brain, Instrumentation Science and Technology, Vol. 35, pp.255-273.

Usakli, A.B. \& Gencer, N.G. (2007). Performance Tests of A Novel Electroencephalographic Data-Acquisition System, The Fifth International Conference on Biomedical Engineering IASTED BIOMED 2007, pp. 253-257, Innsbruck, Austria, February 14-16, 2007.

Usakli, A.B. \& Gurkan S. (2010). Design of a Novel Efficient Human Computer Interface: An Electrooculagram Based Virtual Keyboard, IEEE Transaction on Instrumentation and Measurement, Vol. 59, No.8, pp. 2099-2108. 
Usakli, A.B. (2010). Modeling of Movement-Related Potentials Using a Fractal Approach, Journal of Computational Neuroscience, Vol.28, No. 3, pp. 595-603.

Van Kokswijk, J. \& Van Hulle, M. (2010). Self Adaptive BCI as Service-Oriented Information System for Patients with Communication Disabilities, New Trends in Information Science and Service Science NISS 2010 4th International Conference on, pp. 264-269, Gyeongju, S. Korea, 11-13 May 2010.

Vidaurre, C.; Schlogl, A.; Cabeza, R.; Scherer, R. \& Pfurtscheller, G. (2006). A Fully On-Line Adaptive BCI, Biomedical Engineering, IEEE Transactions on, (June 2006), Vol. 53 6, pp. 1214 - 1219, ISSN: 0018-9294.

Weiskopf, N.; Mathiak, K.; Bock, S.W.; Scharnowski, F.; Veit, R.; Grodd, W.; Goebel, R. \& Birbaumer, N. (2004). Principles of a Brain-Computer Interface (BCI) Based on RealTime Functional Magnetic Resonance Imaging (fMRI), Biomedical Engineering, IEEE Transactions on, (June 2004), Vol. 51 6, pp. 966 - 970, ISSN: 0018-9294.

Wolpaw, J. R (2003). Brain-Computer Interfaces: Signals, Methods, and Goals, Proceedings of the 1st International IEEE EMBS Conference on Neural Engineering, pp. 584-585, Capri Island, Italy, March 20-22, 2003.

Wolpaw, J.; Birbaumer, N.; McFarland, D.; Pfurtscheller, G. \& Vaughan, T. (2002). BrainComputer Interfaces for Communication and Control, Electroencephalography and Clinical Neurophysiology, vol. 113 6, pp. 767-791.

Wolpaw, J.R.; McFarland, D. J. \& Vaughan, T. M. (2000). Brain-Computer Interface Research at the Wadsworth center, IEEE Trans. Rehab. Eng., vol. 8, pp. 222-226.

Wolpaw, J.R.; McFarland, D.J.; Vaughan, T.M. \& Schalk, G. (2003). The Wadsworth Center Brain-Computer Interface (BCI) Research and Development Program, Neural Systems and Rehabilitation Engineering, IEEE Transactions on, (June 2003), Vol.: 112 pp. 1 - 4, ISSN: 1534-4320.

Wu, C.M.; Huang, K.G.; Chang, S.H.; Hsu, S.C. \& Lin, C.L. (2007). EOG Single Switch Morse Code Translate Input Device for Individuals with the Motor Neuron Disease, TENCON 2007 - 2007 IEEE Region 10 Conference, Taipei, Oct. 30-Nov. 22007.

Wu, C.M.; Huang, K.G.; Chang, S.H.; Hsu, S.C. \& Lin, C.L. (2007). EOG Single Switch Morse Code Translate Input Device for Individuals with the Motor Neuron disease, ENCON 2007 - 2007 IEEE Region 10 Conference, Taipei, Taiwan, Oct. 30 2007-Nov. 2 2007.

Yoshiaki, K. (1997). Development of Eye-Gaze Input Interface, Proc. of 7th International conference on Human Computer Interaction, pp.44-49.

Zhai, J.; Barreto, A.; Chin, C. \& Chao, L. (2005). Realization of Stress Detection Using Psychophysiological Signals for Improvement of Human-computer Interactions, Southeast Conference Proceedings, IEEE, pp.415-420, Fort Lauderdale, Florida, USA, 8-10 April 2005.

Zheng, X.; Liu, X. L. J.; Chen, W. \& Hao, Y. (2009). A Portable Wireless Eye MovementControlled Human-Computer Interface for the Disabled, Complex Medical Engineering, 2009. CME. ICME International Conference on, Tempe, Arizona, USA, 911 April 2009. 
AMYOTROPHIC

LATERAL SCLEROSIS

Eaced by Marton H. maver

\section{Amyotrophic Lateral Sclerosis}

Edited by Prof. Martin Maurer
ISBN 978-953-307-806-9

Hard cover, 718 pages

Publisher InTech

Published online 20, January, 2012

Published in print edition January, 2012

Though considerable amount of research, both pre-clinical and clinical, has been conducted during recent years, Amyotrophic Lateral Sclerosis (ALS) remains one of the mysterious diseases of the 21st century. Great efforts have been made to develop pathophysiological models and to clarify the underlying pathology, and with novel instruments in genetics and transgenic techniques, the aim for finding a durable cure comes into scope. On the other hand, most pharmacological trials failed to show a benefit for ALS patients. In this book, the reader will find a compilation of state-of-the-art reviews about the etiology, epidemiology, and pathophysiology of ALS, the molecular basis of disease progression and clinical manifestations, the genetics familial ALS, as well as novel diagnostic criteria in the field of electrophysiology. An overview over all relevant pharmacological trials in ALS patients is also included, while the book concludes with a discussion on current advances and future trends in ALS research.

\section{How to reference}

In order to correctly reference this scholarly work, feel free to copy and paste the following:

Ali Bülent Uşaklı (2012). Human Computer Interactions for Amyotrophic Lateral Sclerosis Patients, Amyotrophic Lateral Sclerosis, Prof. Martin Maurer (Ed.), ISBN: 978-953-307-806-9, InTech, Available from: http://www.intechopen.com/books/amyotrophic-lateral-sclerosis/human-computer-interactions-for-amyotrophiclateral-sclerosis-patients

\section{INTECH}

open science | open minds

\section{InTech Europe}

University Campus STeP Ri

Slavka Krautzeka 83/A

51000 Rijeka, Croatia

Phone: +385 (51) 770447

Fax: +385 (51) 686166

www.intechopen.com

\section{InTech China}

Unit 405, Office Block, Hotel Equatorial Shanghai

No.65, Yan An Road (West), Shanghai, 200040, China

中国上海市延安西路65号上海国际贵都大饭店办公楼 405 单元

Phone: +86-21-62489820

Fax: $+86-21-62489821$ 
(C) 2012 The Author(s). Licensee IntechOpen. This is an open access article distributed under the terms of the Creative Commons Attribution 3.0 License, which permits unrestricted use, distribution, and reproduction in any medium, provided the original work is properly cited. 